\title{
Biodegradable, flame retardant wood-plastic combination via in situ ring-opening polymerization of lactide monomers
}

\author{
Shan $\mathrm{He}^{1}{ }^{1} \cdot$ Yichen $\mathrm{Guo}^{1} \cdot$ Tehila Stone $^{2} \cdot$ Noah Davis $^{3} \cdot$ Dongwhan Kim $^{4} \cdot$ \\ Taejin Kim $^{1} \cdot$ Miriam Rafailovich $^{1}$
}

Received: 26 September 2016/Accepted: 5 November 2016/Published online: 10 January 2017

(C) The Japan Wood Research Society 2017

\begin{abstract}
A wood-plastic combination (WPC) was created via in situ polymerization of the L-lactide monomer (3S)cis-3,6-dimethyl-1,4-dioxane-2,5-dione. Commercial poplar boards (Liriodendron tulipifera) were impregnated with the flame retardant chemical resorcinol bis(diphenyl phosphate)(RDP). These samples were then soaked in a solution of the monomer and deionized water with sulfuric acid $5 \% \mathrm{wt} /$ wood as a catalyst for polymerization. The wood and solution were placed in a vacuum oven for impregnation and polymerization of the monomers. The wood RDP combination was not flame retardant and had an Izod impact strength that was slightly smaller than neat wood sample. Addition of lactide monomer tripled the Izod impact strength relative to wood, and scanning electron microscope (SEM) images indicated that a polymerized coating had formed which reinforced the porous wood structure. Addition of all three components produced a synergy. The Izod impact strength of the material was nearly 14 times greater and the WPC was flame retardant surpassing the stringent UL-94-V0 requirement.
\end{abstract}

Keywords Wood P Plastic Biodegradable Flame retardant

Miriam Rafailovich

miriam.rafailovich@stonybrook.edu

1 Department of Materials Science and Engineering, Stony Brook University, Stony Brook, NY 11794, USA

2 Department of Civil and Environmental Engineering, Princeton University, Princeton, NJ 08544, USA

3 Earl L. Vandermeulen High School, Port Jefferson, NY 11777, USA

4 Smithtown High School West, Smithtown, NY 11787, USA

\section{Introduction}

Wood is a popular building material with commercial applications ranging from construction to furniture manufacturing. While wood is more environmentally compatible than steel or concrete, it is often far weaker in mechanical properties. Additionally, its flammability impedes widespread usage. Wood-plastic combinations (WPCs) have been developed to improve the mechanical and thermal properties of wood. The addition of polymers to wood allows for materials that are simultaneously tough and ductile with less porosity than wood alone. Compacted wood, widespread in today's market, is created by combining wood fibers with polymers; this method has been shown to be highly effective in producing materials stronger than natural wood. However, for some applications it is necessary to treat wood planks and boards rather than their fiber components.

One such method of manufacturing WPCs involves in situ polymerization, which has been successfully performed by Bergman et al. [1] and Khan et al. [2] using acrylic and by Lang et al. [3] using urea monomers. Devi and Maji [4] show that flame retardant additives have been combined with polymerization treatment to create a woodbased material that is strong and flame retardant. A downside to these WPCs is that because of the presence of the newly created polymers, the material is not fully biodegradable. Furthermore, these materials are typically hydrophobic, whereas wood and its constituent fibers are hydrophilic. Therefore, researchers have experimented with in situ polymerization with numerous types of monomers. Yet despite the large variety of WPC reported, none were specifically crafted for flame retardance [5]. In particular, poly (lactic acid) (PLA), a polymer known for its biodegradable properties [6], has been previously 


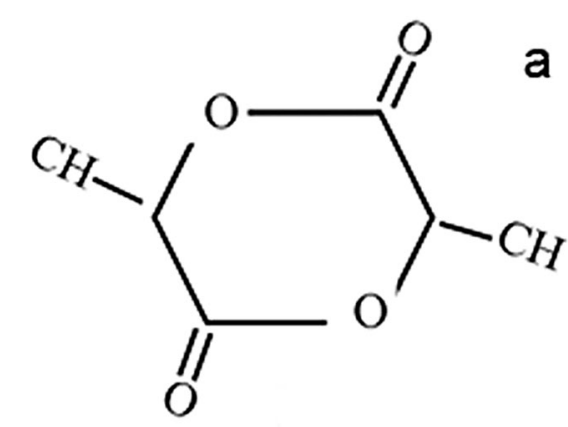

b

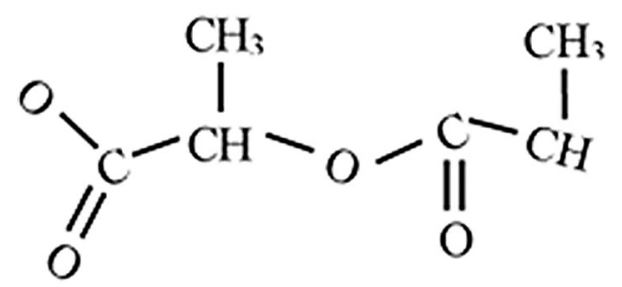

Fig. 1 Diagram of ring-opening polymerization of lactide (a) into poly(lactic acid) (b)

synthesized via in situ polymerization of lactide oligomers. Noel et al. [7, 8] shows that the oligomers, which are larger in size compared to monomers, became entrapped within the wood structure and thus did not fully polymerize.

The research presented here seeks to create a fully biodegradable and fully flame retardant WPC via in situ ring-opening polymerization (Fig. 1) of lactide monomers and impregnation of the flame retardant chemical resorcinol bis(diphenyl phosphate) (RDP). RDP is commercially used as an environmentally safe flame retardant additive and a compatibilizer, and is a more viable option in today's environmentally conscious market than halogen-based flame retardant additives [9-13]. Furthermore, it has also been shown that phosphates can effectively react with cellulosic materials such as cotton to form flame retardant cloths. Therefore, it is reasonable to propose that RDP interact with a biodegradable monomer and wood to form a flame retardant, biodegradable WPC. Here, we report on the fabrication of a lactide wood RDP combination to achieve a WPC with enhanced mechanical, as well as thermal properties

\section{Materials and methods}

\section{Materials}

Commercial poplar boards (Liriodendron tulipifera) were bought from Home Depot cut into eight $12.0 \mathrm{~cm} \times 12.0 \mathrm{~cm} \times 0.5 \mathrm{~cm}$ pieces. This type of wood was chosen since it is easily available in many consumer outlets, and we believe that the process is generally applicable to all types of wood. The specific gravity and the Izod impact strength, $I$, of the wood was measured and found to be, specific gravity $=0.47$ and $I=42.5 \pm 5.0 \mathrm{~kJ} / \mathrm{m}^{2}$. This value is in good agreement with that reported for this type of wood, $I=44.97 \mathrm{~kJ} / \mathrm{m}^{2}$, at a specific gravity of 0.49 , and a moisture content of $12 \%$ [14].

The L-lactide monomer (3S)-cis-3,6-dimethyl-1,4-dioxane-2,5-dione was acquired from Sigma Aldrich Co. LLC. Sulfuric acid $98 \%$ from J.T. Baker Chemicals was used as a catalyst for polymerization. Fyrolflex RDP from ICL Industrial Products was used as a flame retardant chemical additive.

\section{Impregnation of RDP}

Four wood pieces were soaked in approximately $360 \mathrm{~mL}$ of RDP for $24 \mathrm{~h}$ in standard conditions. The wood samples were then washed and weighed; each sample contained approximately 5\% wt/wood RDP. Two of these pieces were set aside for monomer impregnation and polymerization

\section{Impregnation of monomers}

Two solutions of $12.5 \mathrm{~g}$ of lactide monomers soaked in $360 \mathrm{~mL}$ of deionized water each were heated for an hour to increase solubility. $2 \mathrm{~mL}$ of sulfuric acid were added to each solution. One solution was used for two pure wood samples and the other solution was used for the two wood samples that were impregnated with RDP. The wood pieces and solution (Fig. 2) were then placed in a vacuum for $2 \mathrm{~h}$ at room temperature followed by $12 \mathrm{~h}$ in standard conditions.

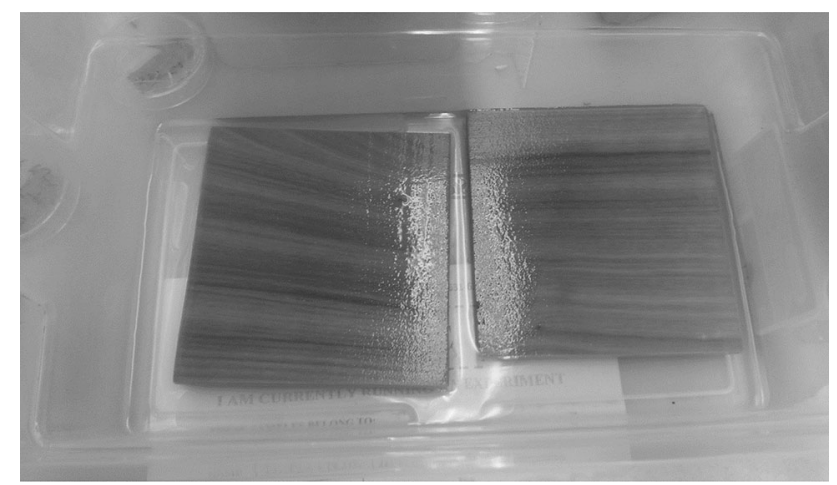

Fig. 2 Wood samples soaked in monomer solution preceding vacuum impregnation and heat polymerization 


\section{Polymerization and dehydration}

After impregnation, the wood pieces were placed in an oven at $120{ }^{\circ} \mathrm{C}$ for $1 \mathrm{~h}$-the minimum time and temperature required for polymerization [7]. The samples were then washed and weighed; it was concluded that each sample contained $15 \% \mathrm{wt} /$ wood lactide on average. It was observed that the wood was softened, most likely due to the presence of sulfuric acid. Thus, the wood samples were dehydrated by heating for $96 \mathrm{~h}$ at $80-100{ }^{\circ} \mathrm{C}$, a process that has been performed in previous research [8]. In case the RDP was evaporated during this extensive heating period, the two wood samples that were initially soaked in RDP preceding monomer impregnation were soaked again for $24 \mathrm{~h}$ in $360 \mathrm{~mL}$ of RDP in standard conditions.

\section{Investigation of properties}

After the above processes were performed, the resulting wood materials were two control samples; two samples with wood and RDP; two samples with wood and lactide; and two samples with wood, lactide and RDP (Fig. 3). Each of these samples were subsequently cut to fit the parameters of the tests to be performed.

\section{Mechanical properties}

The impact toughness values of the wood and WPCs were investigated using the TMI Izod Impact ASTM D256, ISO 180 with units set at $\mathrm{kJ} / \mathrm{m}^{2}$ [15]. The densities of the samples were also investigated by measuring the dimensions of each specimen with a caliper and the mass with a scale.

\section{Thermal properties}

To determine the flame retardant capabilities of the WPCs, the UL-94 Vertical Flame Test was performed. Burned samples were preserved for subsequent testing of chemical composition and structure.

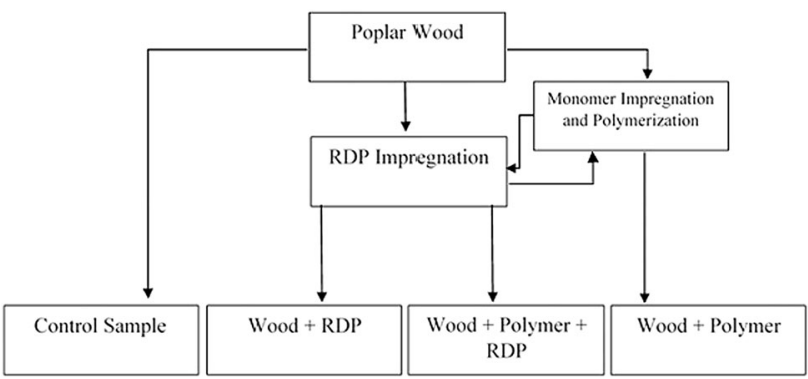

Fig. 3 Outline of procedures used to manufacture the wood-plastic combination (WPC) and the subsequent four materials formed

\section{Chemical composition}

Fourier Transform Infrared Spectroscopy (FTIR) was used via a Thermo Scientific Nicolet iS 10 spectrometer system to collect the spectra of the samples. Each spectrum was investigated for wavelengths of bonds that would indicate the presence of PLA and RDP, as well as variations in intensity of certain peaks that would elude to grafting of PLA with wood functional groups. In particular, the spectrum of the wood/PLA/RDP combination was analyzed for information on the interaction between the three components of the material.

Scanning Electron Microscopy (SEM) and Electron Dispersive X-ray Spectroscopy (EDX) were used at a $300 \mathrm{X}$ magnification to observe the structure of the wood and WPCs before and after samples were burned in the UL-94 test. EDX measures the X-ray spectra from elements excited by the electron beam and can be used to provide an elemental map of the sample components imaged with the SEM. Particular attention was given to the wood/PLA/RDP combination to observe polymerization or entrapment of PLA and RDP.

\section{Results and discussion}

\section{Mechanical and structural properties}

After testing and recording the impact toughness values for each sample (Fig. 4), it was observed that the wood/RDP combination had slightly decreased toughness values compared to the control. This reduction was expected, as the reaction with phosphates was shown to embrittle cellulosic materials [16]. Reacting the wood with lactide monomer to form the wood/lactide combination nearly

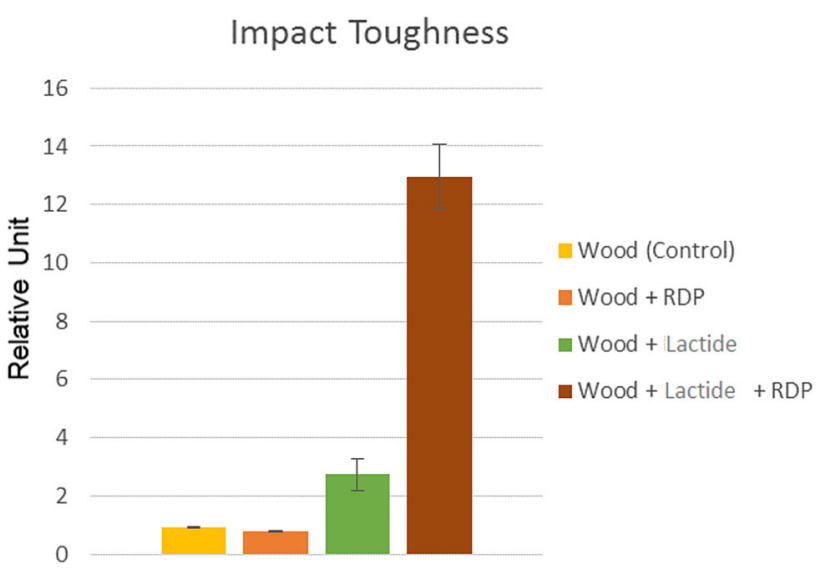

Fig. 4 Impact toughness values of control wood, wood treated with resorcinol bis(diphenyl phosphate)(RDP), and the two wood-plastic combination (WPC) samples where the value for the control sample was $I=42.5 \pm 5.0 \mathrm{~kJ} / \mathrm{m}^{2}$ 


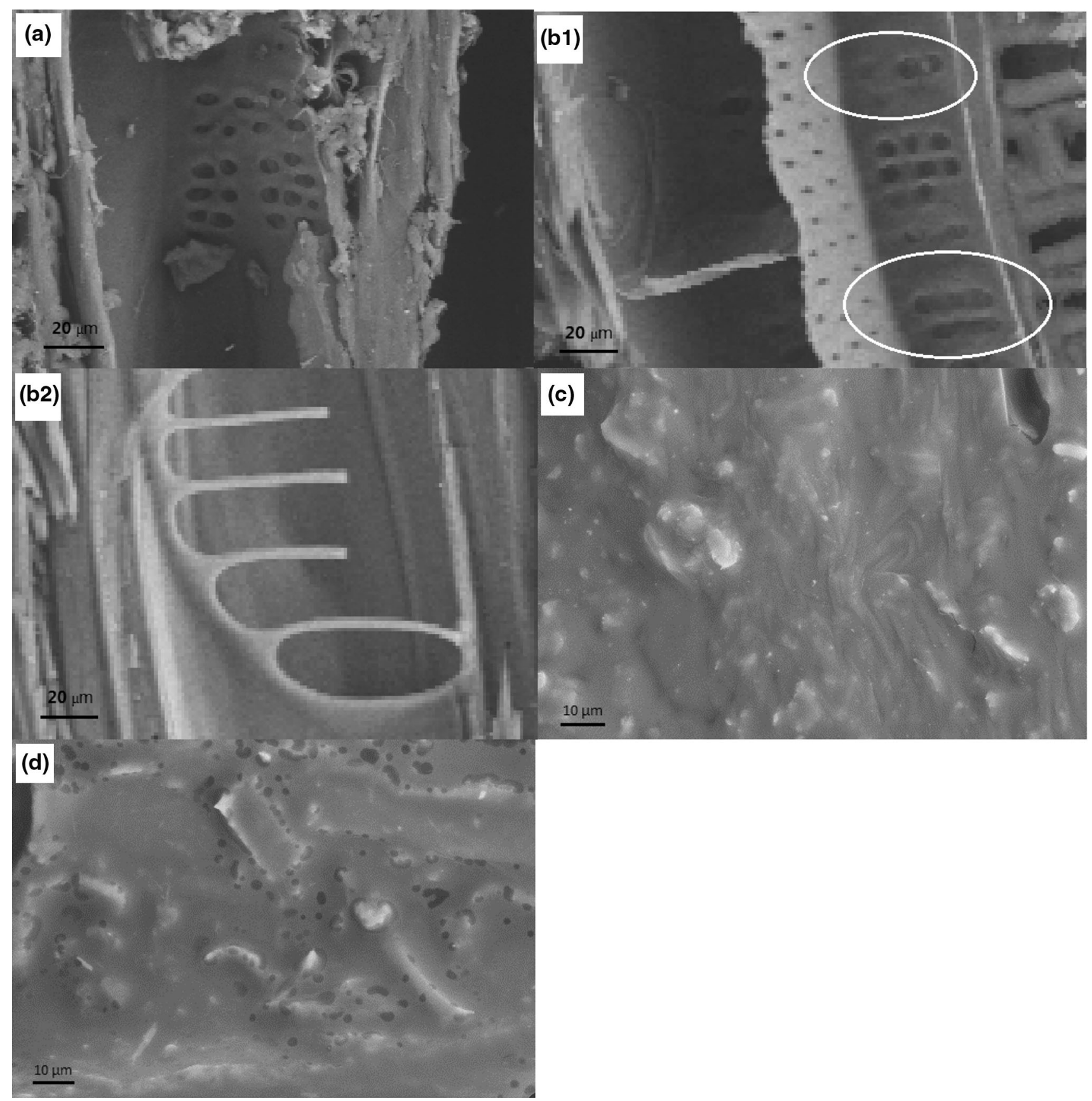

Fig. 5 Scanning electron microscope (SEM) image of a wood, b1, b2 wood with lactide, $\mathbf{c}$ wood with resorcinol bis(diphenyl phosphate) (RDP), and d wood with lactide and resorcinol bis(diphenyl phosphate) (RDP)

tripled the impact toughness relative to the untreated sample. This result was surprising since previous studies of WPCs produced by monomer polymerization enhanced the moduli, but at the expense of the impact toughness, which decreased by $40 \%$ or more [17]. Incorporation of the monomer usually fills in the wood pores, thus increasing the compression strength, but at the expense of decreasing the ability of the wood to store the energy of an impact. In this case, the increase of the impact toughness indicates that the monomer has polymerized within the cavities of the wood strengthening the interface between the two materials and forming a combination that is both resistant to fracture, as well as shock absorbing.

The wood/RDP/lactide combination provided the most dramatic results, as the combination of these substances created a material that was up to 14 times stronger than the control. Not only is an increase in toughness of such magnitude rare in wood treatment; it is a particularly rare 
(a)

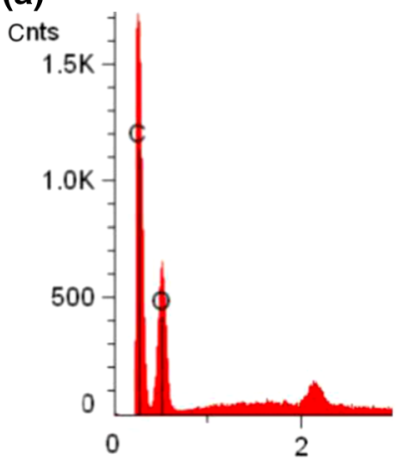

(b)

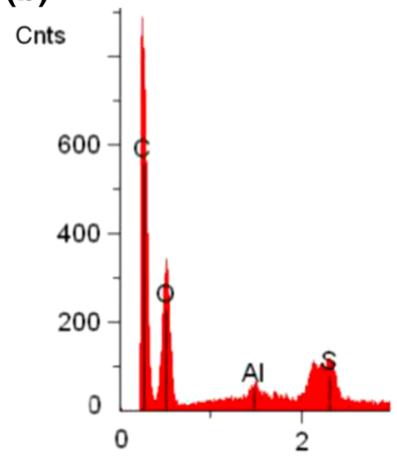

(c)

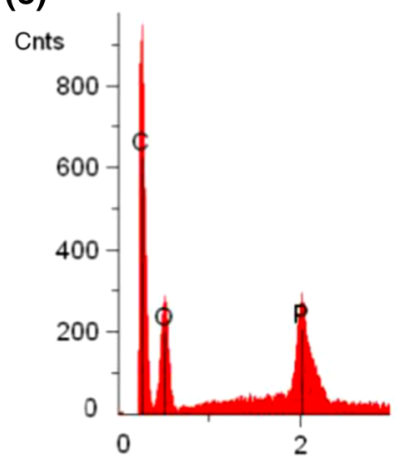

(d)

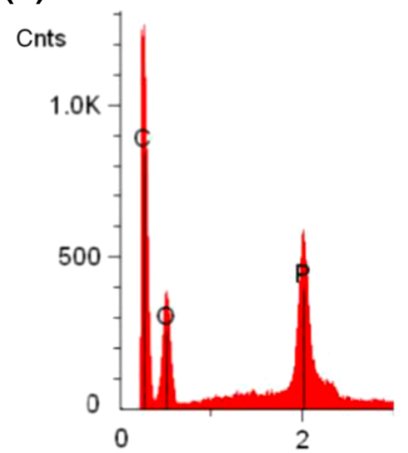

Fig. 6 Electron dispersive X-ray (EDX) spectra of a wood, b wood with lactide, c wood with resorcinol bis(diphenyl phosphate) (RDP), and d wood with lactide and resorcinol bis(diphenyl phosphate) (RDP)

Table 1 UL-94 flame test ratings

\begin{tabular}{llll}
\hline Sample & T1 & T2 & Rating \\
\hline Wood (control) & $>30$ & N/A & Fail \\
Wood + RDP & $>30 ;<120$ & N/A & Fail \\
Wood + lactide & $>30 ;<120$ & N/A & Fail \\
Wood + RDP + lactide & $\sim 1$ & $<1$ & V-0 \\
\hline
\end{tabular}

$\mathrm{T} 1$ and $\mathrm{T} 2$ refers to the time in seconds until the sample self-extinguished after initial and secondary exposure, respectively, to a $2 \mathrm{~mm}$ flame

$R D P$ resorcinol bis(diphenyl phosphate)

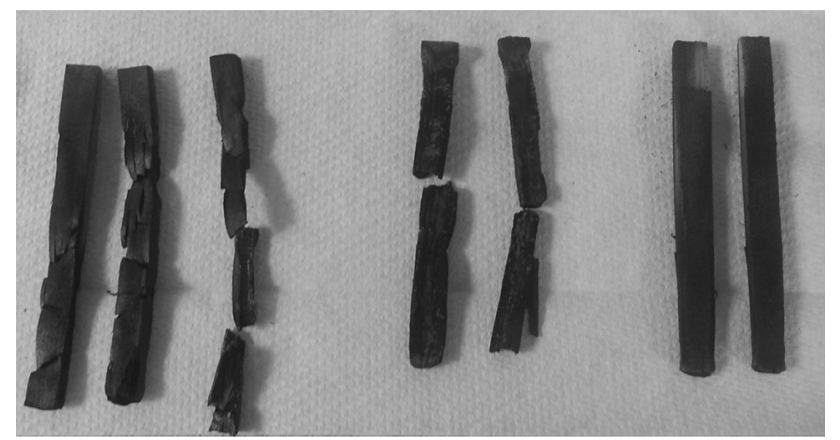

Fig. 7 Treated samples after conducting the UL-94 Flame Test, from left: 3 wood/PLA samples, 2 wood/resorcinol bis(diphenyl phosphate) (RDP) samples, and 2 wood/resorcinol bis(diphenyl phosphate) (RDP)/PLA samples. The minor discoloration at the top ends of the wood/resorcinol bis(diphenyl phosphate)(RDP)/PLA composites resulted from polymerization; it is unrelated to the flame test

result with flame retardant treatments in general [5]. Addition of flame retardant compounds in polymer produced strongly phase segregated domains. The inclusions will increase the modulus of the materials, as has been reported, but the addition of interfaces within the material increases the ease of crack propagation and decreases the Izod impact. In this case the large increase in Izod impact when all three components are present is indicative of a

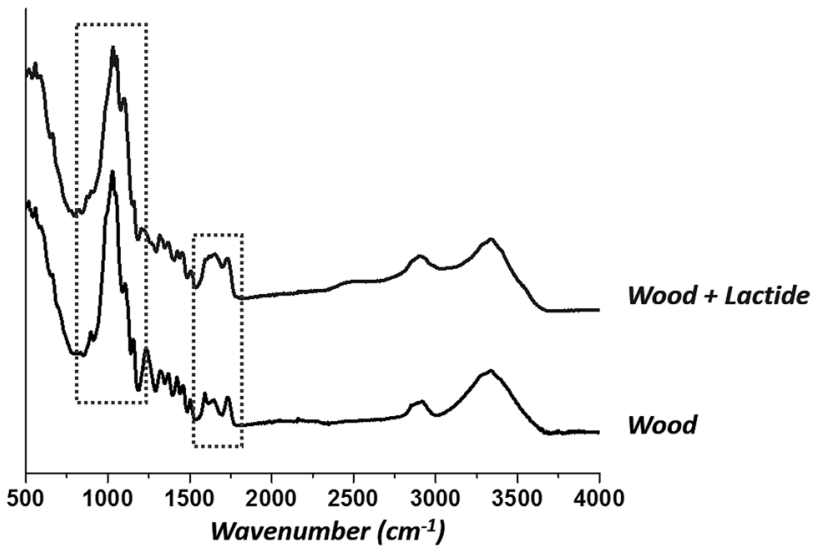

Fig. 8 Fourier transform infrared spectroscopy (FTIR) spectrum of the wood/lactide composite compared to the control wood spectrum

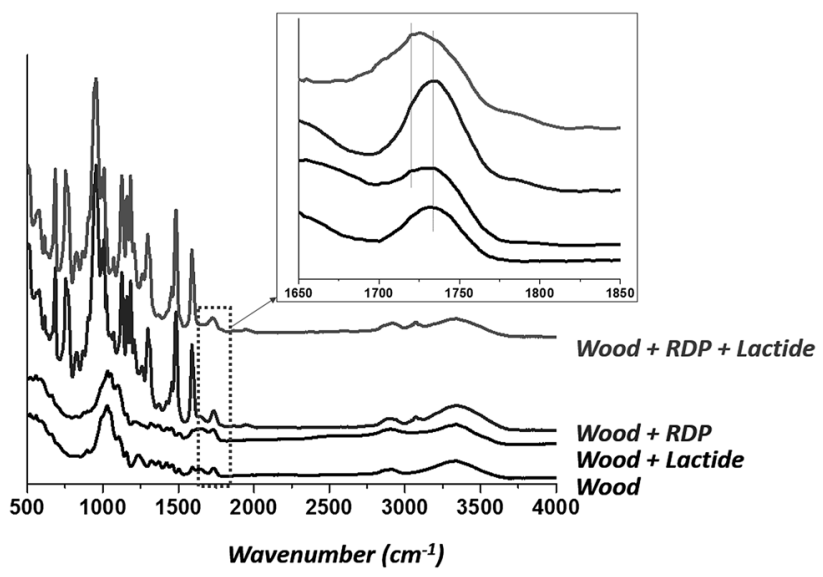

Fig. 9 Fourier transform infrared spectroscopy (FTIR) spectra of the four wood and wood-plastic combination (WPC) samples

sudden increase in interfacial adhesion which blocks propagation of the crack upon impact. RDP is a well known plasticizer, which, when added to polymers, will decrease the number of entanglements, decrease the modulus and degrade the mechanical properties, as observed in Fig. 4. 
On the other hand, when added to the PLA/Wood combination it can compatibilize the materials by increasing their interface thereby strengthening the material and increasing the resistance to crack propagation.

The internal structure of WPC can be determined by SEM images of fracture surfaces and associated EDX spectra of the chemical compositions. In Fig. 5a, we show the image of the unmodified wood, where one can see the typical porous structures formed by the cell and lumen. In Fig. 5b1, we show the image obtained from fracture of the samples loaded with monomer and polymerized in the presence of sulfuric acid. From the figure we can clearly see that a membrane had polymerized from the oligomer, which covers the pores in the wood. It is interesting to note that for the sample shown in Fig. 5b2, polymer fibrils are observed which span across the fracture surface, and mitigated the energy deposited by a crack.

In Fig. 5c, we show the images of the WPC where only RDP was added, where we can see that a thick monolayer was deposited. The monolayer appears to be well dispersed and from EDX spectra (Fig. 6) contains large amount of phosphorous indicating that the RDP is present at the sample surface. A similar image shown in Fig. 5d was from the samples where all three components were present, consisted with the RDP improving the compatibilization and strengthening the interfaces.

\section{Flame retardance}

The UL-94 Vertical Flame Test was performed on all four samples (Table 1). It was observed that the addition of RDP alone, as well as the addition of lactide alone caused the wood to self-extinguish, but not within the $30 \mathrm{~s}$ requirement of the UL-94 rating system. In contrast, the control wood sample did not self-extinguish. The WPC containing both RDP and lactide was the only sample to pass the flame test. This combination achieved the highest rating of $\mathrm{V}-0$, self-extinguishing in $1 \mathrm{~s}$ or less. Additionally, while the other two non-control samples were burned throughout and showed signs of breakage, the wood/RDP/ lactide sample exhibited minor charring only at the bottom of the sample, which was the point of flame exposure, and remained fully intact (Fig. 7). These results further prove the possibility of an interaction between RDP and lactide in situ, as the combination of these two materials within wood creates an extremely flame retardant material.

\section{Chemical composition and characterization}

To prove the existence of a novel interaction between RDP and lactide and to examine possible causes of this interaction, FTIR and SEM analyses were performed on the samples. After comparing the spectra of the control sample and the wood/lactide combination (Fig. 8), an increase in the size of the $\mathrm{C}-\mathrm{O}$ stretching peak at $1150 \mathrm{~cm}^{-1}$ in the combination sample indicates an increase in carboxyl groups. Additionally, the appearance of a peak at $1650 \mathrm{~cm}^{-1}$ shows the presence of carboxylic acid in the form of amides, most likely due to the use of sulfuric acid as a catalyst. Both of these aforementioned peaks show that lactide is grafted with the wood cell walls, as the carbonyl groups in lactide most likely bonded with the hydroxyl groups of the wood to form carboxylic acids. Upon examination of the spectra of all four samples (Fig. 9), it was noted that all spectra except for that of the wood/ lactide/RDP combination contained a peak at $1735 \mathrm{~cm}^{-1}$, representing ester groups. On the other hand, the wood/ lactide/RDP spectrum showed a shift in this peak to $1720 \mathrm{~cm}^{-1}$, which represents the presence of aliphatic compounds. Furthermore, the peak at $3400 \mathrm{~cm}^{-1}$, corresponding to hydroxyl groups, decreased in intensity in the wood/RDP/lactide sample, showing that this sample grafted with the wood cell walls and thus decreased the concentration of hydroxyl groups. These differences in spectrum peaks show that the addition of both RDP and lactide cause a unique reaction inside the wood structure compared to adding RDP or lactide alone. However, more research needs to be done to reach a conclusion on the precise chemical characteristics of the wood/RDP/lactide material and what reactions caused its mechanical and thermal properties.

\section{Conclusions}

We have constructed a WPC composed of wood, PLA, and RDP. Impregnation of wood with only RDP did not yield a flame retardant WPC, and the Izod impact slightly degraded relative to the neat wood sample. Addition of lactide monomer tripled the Izod impact relative to wood, and SEM images indicated that a polymerized membrane had formed reinforcing the porous wood structure. Addition of all three components produced a synergy. The Izod impact of the material was nearly 14 times greater and the WPC was flame retardant surpassing the stringent UL-94-V0 requirement.

Acknowledgements We would like to thank the NSF-INSPIRE program (Grant \#1344267). We would also like to thank the Advanced Energy Center for access to the ThINC facility.

\section{Compliance with ethical standards}

Conflict of interest The authors declare that they have no conflict of interest.

This article does not contain any studies with human participants or animals performed by any of the authors. 


\section{References}

1. Bergman R, Ibach R, Lapasha C, Denig J (2009) Evaluating physical property changes for small-diameter, plantation-grown southern pine after in situ polymerization of an acrylic monomer. For Prod J 59(10):64-71

2. Mubarak A, Khan KM, Idriss Ali, Jahan MS (1999) Characterization of wood and wood-plastic composite. Polym-Plast Technol 38(4):753-765

3. Lang Q, She Y, Chen H, Pu J (2013) Poplar wood-methylolurea composites prepared by in situ polymerization. J Appl Polym Sci 130(2):933-938

4. Devi R, Maji T (2013) In situ polymerized wood polymer composite: effect of additives and nanoclay on the thermal, mechanical properties. Mater Res 16(4):954-963

5. Li Y (2011) Wood-polymer composites. In: Tesinova P (ed) Advances in composite materials - analysis of natural and manmade materials. ISBN: 978-953-307-449-8 InTech. http://www. intechopen.com/books/advances-in-composite-materials-analysis -of-natural-and-man-madematerials/wood-polymer-composites. Accessed 09 Sep 2011

6. Ren J (2010) Biodegradable poly (lactic acid) synthesis, modification, processing and applications. Tsinghua University Press, Beijing

7. Noël M, Fredon E, Mougel E, Masson D, Masson E, Delmotte L (2009) Lactic acid/wood-based composite material part 1: synthesis and characterization. Bioresour Technol 100:4711-4716

8. Noël M, Mougel E, Fredon E, Masson D, Masson E (2009) Lactic acid/wood-based composite material part 2: physical and mechanical performance. Bioresour Technol 100:4717-4722

9. Pawlowski K, Schartel B (2007) Flame retardancy mechanisms of triphenyl phosphate, resorcinol bis(diphenyl phosphate) and bisphenol a bis(diphenyl phosphate) in polycarbonate/acrylonitrile-butadiene-styrene blends. Polym Int 56:1404-1414

10. Guo Y, Yang K, Zuo X, Xue Y, Marmorat C, Liu Y, Chang C, Rafailovich M (2016) Effects of clay platelets and natural nanotubes on mechanical properties and gas permeability of poly (lactic acid) nanocomposites. Polymer 83:246-259

11. Guo Y, He S, Yang K, Xue Y, Zuo X, Yu Y, Liu Y, Chang C, Rafailovich M (2016) Enhancing the mechanical properties of biodegradable polymer blends using tubular nanoparticle stitching of the interfaces. ACS Appl Mater Interfaces 8(27): 17565-17573

12. Pack S, Kashiwagi T, Cao C, Korach C, Lewin M, Rafailovich M (2010) Role of surface interactions in the synergizing polymer/clay flame retardant properties. Macromolecules 43(12):5338-5351

13. Pack S, Bobo E, Muir N, Yang K, Swaraj S, Ade H, Cao C, Korach C, Kashiwagi T, Rafailovich M (2012) Engineering biodegradable polymer blends containing flame retardant-coated starch/nanoparticles. Polymer 53:4787-4799

14. Bao F, Fu F, Choong E, Hse C (2001) Contribution factor of wood properties of three poplar clones to strength of laminated veneer lumber. Wood Fiber Sci 33(3):345-352

15. ASTM D256: Standard test methods for determining the Izod pendulum impact resistance of plastics. ASTM International, West Conshohocken, PA, vol 08.01

16. Reid D, Mazzeno L (1949) Preparation and properties of cellulose phosphates. Ind Eng Chem 41(12):2828-2831

17. Li Y, Liu Y, Wang X, Wu Q, Yu H, Li J (2011) Wood-polymer composites prepared by the in situ polymerization of monomers within wood. J App Polym Sci 119:3207-3216 